\title{
Biomassa aérea e NDVI em zona ecotonal cerrado-caatinga da Flona de Palmares, Altos, Piauí, Brasil
}

\begin{abstract}
A estimativa da biomassa é uma forma de avaliar a quantidade de material biológico de uma floresta quanto à sua conversão de energia e ciclagem de nutrientes, como também estudos referentes à biomassa vegetal e à composição nutricional das plantas podem compor um importante banco de dados para o desenvolvimento de programas de conservação. A vegetação aérea exerce uma importante função na avaliação de um ecossistema florestal. Diante disso o estudo teve como objetivo quantificar a biomassa da vegetação aérea e analisar a sua relação com o NDVI da Floresta Nacional de Palmares, Altos, Piauí. O estudo fo realizado em uma unidade de conservação de uso sustentável, a Floresta Nacional dos Palmares está localizada na Zona Rural do município de Altos/PI, tendo como limite a capital Teresina. Foram considerados nessa pesquisa apenas os indivíduos que estavam dentro das parcelas de $20 \mathrm{~m} \times 20 \mathrm{~m}$, coletadas em 09 glebas, totalizando uma área de 168 ha. Foram incluídos nesse estrato todos os indivíduos com a circunferência na altura do peito (CAP) igual ou superior a $15 \mathrm{~cm}$. Com o NDVI foi possível ter uma visualização da vegetação da Flona, e assim fazer uma comparação com os resultados da biomassa, que foram coerentes com a imagem do NDVI. A biomassa total em árvores vivas e mortas na Flona de Palmares foi estimada em $313.050 \mathrm{Mg} / \mathrm{ha}$, no período seco de 2019 .
\end{abstract}

\section{Aerial biomass and NDVI in a cerrado-caatinga ecotonal zone at Flona Palmares, Altos, Piaui, Brazil}

\begin{abstract}
Biomass estimation is a way of assessing the amount of biological material in a forest in terms of its energy conversion and nutrient cycling, as well as studies on plant biomass and the nutritional composition of plants can compose an important database for the development of conservation programs. Therefore, the study aimed to quantify the biomass of aerial vegetation and analyze its relationship with the NDVI (Normalized Difference Vegetation Index) of the Palmares National Forest, Altos, Piauí. The study was carried out in a sustainable use conservation unit, the Palmares National Forest is located in the Rural Zone of the municipality of Altos/PI, having as its limit the capital Teresina. In this research, only individuals who were within the $20 \mathrm{~m} \times 20 \mathrm{~m}$ plots, collected in 09 plots, totaling an area of $168 \mathrm{ha}$, were considered. In this stratum, all individuals with a circumference at breast height (CAP) equal to or greater than $15 \mathrm{~cm}$ were included. With the NDV it was possible to have a visualization of the Flona vegetation, and thus make a comparison with the biomass results, which were consistent with the NDVI image. The total biomass in live and dead trees in Flona de Palmares was estimated at 313,050 Mg/ha, in the dry period of 2019.
\end{abstract}

Keywords: Caatinga biome; Conservation unit; Nutriente cycling.

Maria Letícia Stefany Monteiro Brandão (iD) Instituto Federal do Piauí, Brasil http://lattes.cnpq.br/9624239072922218 http://orcid.org/0000-0001-6488-1300 marialeticia.gab@gmail.com

Bruna de Freitas Iwata (D)

Instituto Federal do Piauí, Brasil

http://lattes.cnpq.br/3036032785449787 http://orcid.org/0000-0002-6465-9731

iwata@ifpi.edu.br

Gaspar da Silva Alencar (iD

Instituto Chico Mendes de Conservação da Biodiversidade, Brasil

http://lattes.cnpq.br/8613386234495497

http://orcid.org/0000-0002-2764-1516

gaspargeografo343@gmail.com
Suzane Pereira Carvalho (iD

Instituto Federal do Piauí, Brasil

http://lattes.cnpq.br/0681405536125095 http://orcid.org/0000-0003-4366-6868 suzy.p.16@hotmail.com

Karoline de Sousa Almeida (iD)

Instituto Federal do Piauí, Brasil

http://lattes.cnpq.br/5030224857387831

http://orcid.org/0000-0003-2436-4856

karoline.almeida09@hotmail.com

Camila Maria Alves da Silva (it)

Instituto Federal do Piauí, Brasil

http://lattes.cnpq.br/5176777947501187

http://orcid.org/0000-0002-6125-1108

camilaangelo3015@gmail.com
Israel Lobato Rocha (iD)

Instituto Federal do Piauí, Brasil

http://lattes.cnpq.br/6518724518793037 http://orcid.org/0000-0002-4496-9935 israel.lobato@ifpi.edu.br

Mauro César de Brito Sousa (iD

Instituto Federal do Piauí, Brasil

http://lattes.cnpq.br/2049460389729603

http://orcid.org/0000-0002-0650-6577

mauro.sousa@ifpi.edu.br 


\section{INTRODUÇÃO}

As FLONA's (Florestas Nacionais) segundo a Lei Federal n 9.985 de 18 de julho de 2000 (SNUC), são áreas florestais compostas principalmente por espécies nativas, que tem como objetivo conciliar a conservação e o uso sustentável dos recursos naturais das florestas, com incentivos ao desenvolvimento de pesquisas científicas que busquem o aprimoramento de técnicas de manejo sustentável.

Além da importância econômica das áreas florestais e seu grande valor na manutenção da biodiversidade de espécies da fauna e flora, as florestas possuem também grande capacidade de acumular biomassa e estão entre os ecossistemas terrestres que apresentam a maior quantidade de biomassa por unidade de área (FIORENTIN et al., 2015). Sendo também utilizada por muitos países em desenvolvimento como fonte de energia (SALVADOR et al., 2016).

A estimativa da biomassa é uma forma de avaliar a quantidade de material biológico de uma floresta quanto à sua conversão de energia e ciclagem de nutrientes, como também estudos referentes à biomassa vegetal e à composição nutricional das plantas podem compor um importante banco de dados para o desenvolvimento de programas de conservação, e ser um referencial comparativo para a recuperação de áreas degradadas e para a administração de remanescentes florestais, combatendo o uso indiscriminado da floresta (SILVA, 2019).

O conhecimento sobre o estoque de biomassa florestal em diferentes tipologias fornece informações para o direcionamento das decisões que possam contribuir para a conservação das florestas (ZIEMMER et al., 2016). Fiorentin et al. (2015) destacam que a biomassa florestal tem sido estudada com diversos propósitos, entre os quais está a ciclagem de nutrientes, para fins energéticos e na avaliação de crescimento de florestas.

As estimativas de biomassa disponíveis na literatura vêm de estudos que se utilizam de métodos diretos e indiretos. Segundo Sanquetta et al. (2014), o método direto consiste na derrubada e pesagem de todas as árvores que ocorrem em parcelas fixas, fornecendo estimativas. No método indireto, as estimativas são feitas a partir de dados de inventários florestais e no emprego de fatores ou equações, sendo o volume da madeira, a principal variável. Neste método, a biomassa é estimada a partir do volume da madeira, usando-se a densidade média da madeira e um fator de correção para árvores.

A vegetação aérea exerce uma importante função na avaliação de um ecossistema florestal. Diante disso o estudo teve como objetivo quantificar a biomassa da vegetação aérea e analisar a sua relação com o NDVI (Normalized Difference Vegetation Index) da Floresta Nacional de Palmares, Altos/PI.

\section{MATERIAIS E MÉTODOS}

\section{Área de estudo}

O estudo foi realizado em uma unidade de conservação de uso sustentável, a Floresta Nacional dos Palmares está localizada na Zona Rural do município de Altos/PI, tendo como limite a capital Teresina (Figura 1). A Flona abrange uma área de $170 \mathrm{ha}$, uma área relativamente pequena considerando seus objetivos como 
de exploração e manejo dos recursos florestais.

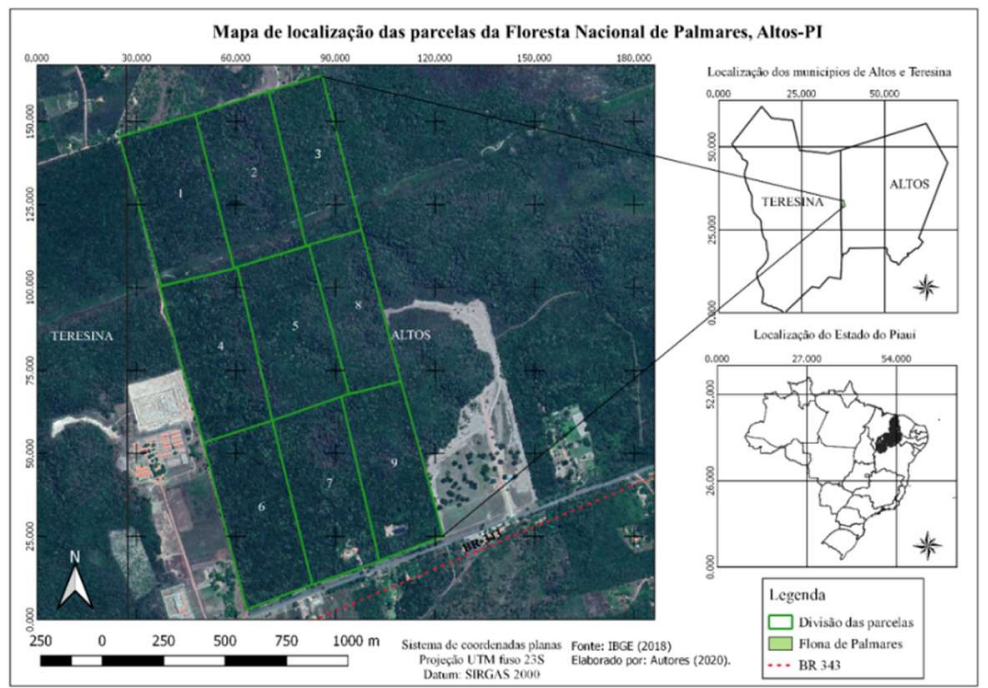

Figura 1: Mapa de localização e divisão das parcelas na Floresta Nacional de Palmares, Altos/PI.

A temperatura média anual do município de Altos-PI onde está localizada a Flona é de $28^{\circ} \mathrm{C}$ e o índice médio pluviométrico anual é de $1200 \mathrm{~mm}$. O clima da região na qual foi realizado o estudo enquadra-se no tipo Aw de acordo com a classificação de Köppen e Geiger, o clima é tropical, apresentando chuva mal distribuída ao longo do ano, e chuvas de verão. Na Figura 2, são apresentados os dados de precipitação e temperatura média do ar da Estação Meteorológica de Teresina/PI, a distância da estação meteorológica para a Flona é de $24 \mathrm{~km}$.

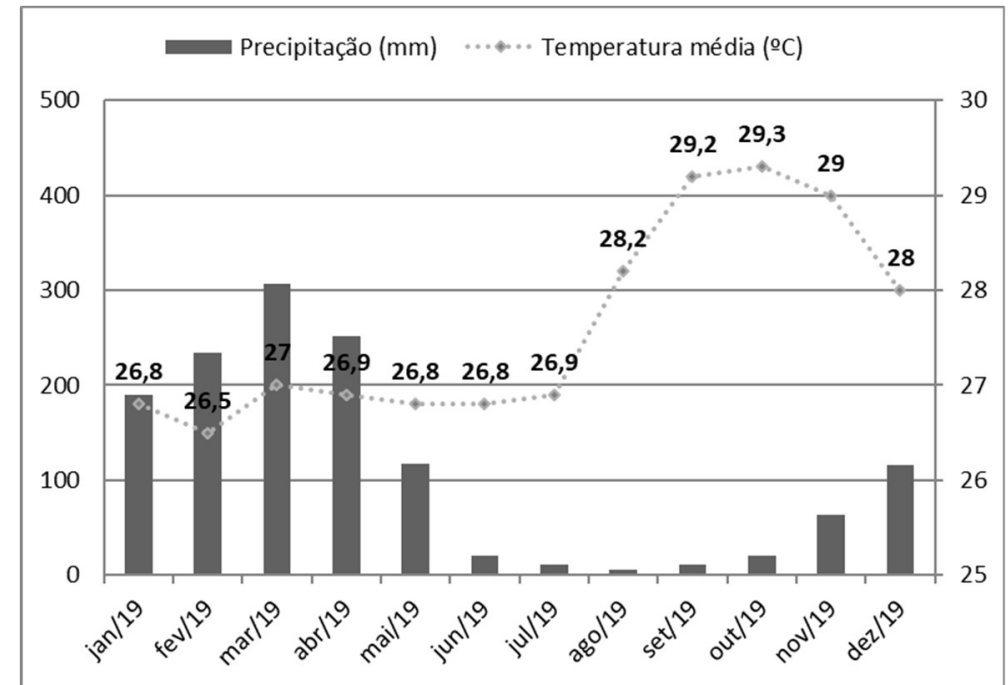

Figura 2: Dados de precipitação e temperatura média do ar da estação meteorológica automática de Teresina/PI.

Fonte: INMET, 2019.

A vegetação da Floresta Nacional de Palmares é representada por uma área de tensão ecológica entre caatinga e mata dos cocais com floresta semidecídua e manchas de cerrado. Segundo inventários fitossociológicos já conduzidos na Flona, existem indivíduos, distribuídos entre 63 (sessenta e três) espécies de plantas e 26 (vinte e seis) famílias botânicas das quais as mais representativas na área são da família Fabaceae (SOUSA et al., 2018).

A vegetação da FLONA Palmares é constituída, quase em sua totalidade, por indivíduos de porte 
arbóreo e arborescente, constituídos basicamente de árvores acima de $5 \mathrm{~m}$ de altura. Ainda segundo Sousa et al. (2018). A situação fitogeográfica de transição e contato, a que está condicionada a FLONA de Palmares, confere a essa unidade uma diversidade de espécies vegetais nativas, representativas dos principais domínios fitogeográficos brasileiros. São encontradas mais de 40 (quarenta) espécies de plantas que são nativas do Domínio Amazônico, embora muitas destas façam parte da formação vegetal de quatro outros domínios, o Cerrado, a Caatinga, a Mata Atlântica e Pantanal.

\section{Coleta de dados da biomassa vegetal aérea e cálculos da biomassa vegetal arbórea}

Os dados sobre a vegetação da biomassa aérea foram coletados a partir de visitas a campo (Figura 3 A, 3 B). Foram considerados nessa pesquisa apenas os indivíduos que estavam dentro das parcelas de $20 \mathrm{~m} \mathrm{x}$ $20 \mathrm{~m}$, coletadas em 09 (nove) glebas conforme mostrado na figura 1, totalizando uma área de $168 \mathrm{ha} .0$ material utilizado foi um GPS (Global Position System) de navegação para marcar o perímetro das parcelas, uma trena digital para medir a altura dos indivíduos e outro manual para medir o CAP (circunferência na altura do peito) e uma planilha de campo que foi considerado as seguintes informações: as características da espécie e distanciamentos (CAP e altura) e as condições fitossanitárias (vitalidade, injúrias mecânicas e infestação). Foram incluídos nesse estrato todos os indivíduos com a circunferência na altura do peito (CAP) igual ou superior a $15 \mathrm{~cm}$.
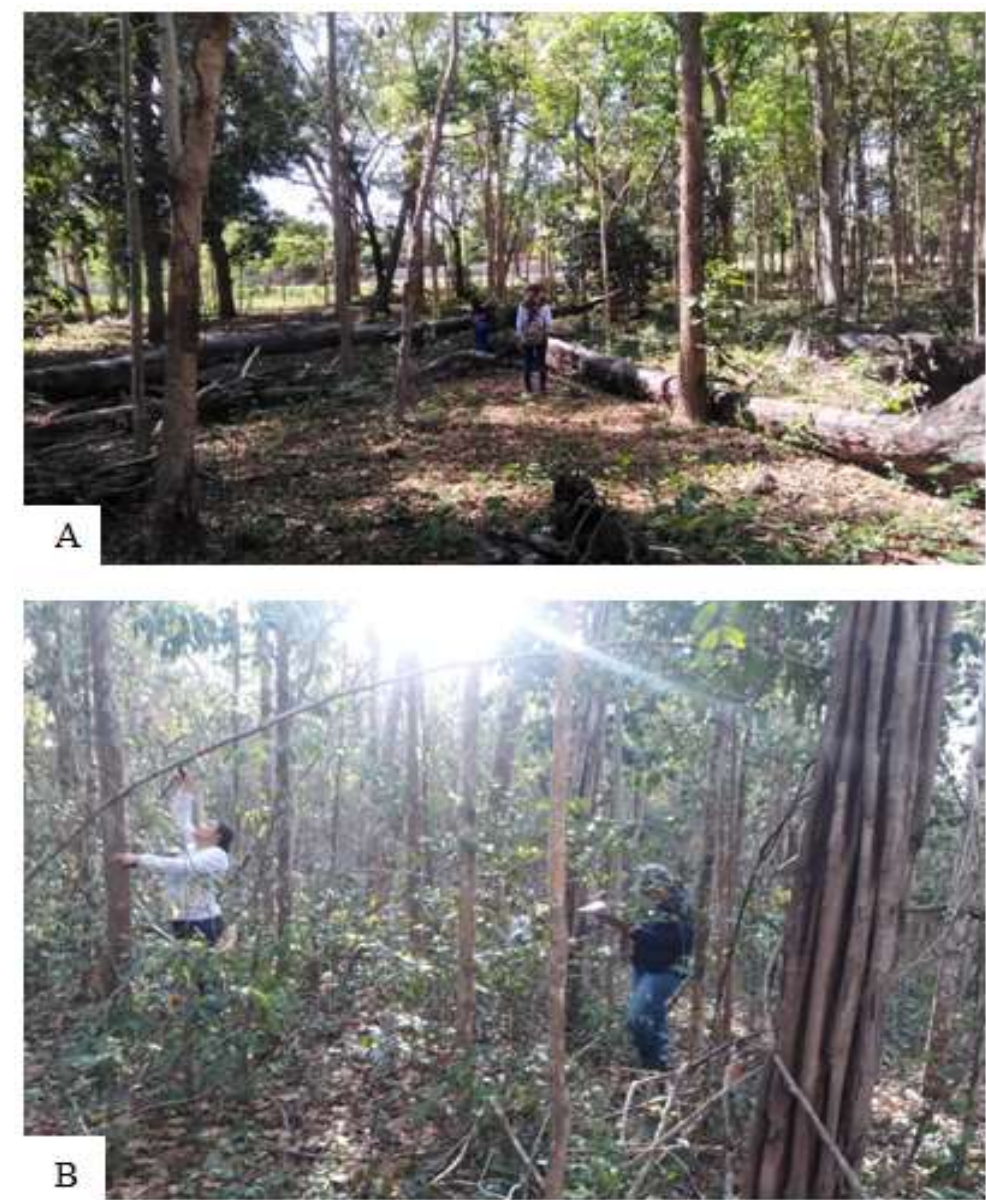

Figura 3: (A) Coleta de dados dos indivíduos mortos/caídos. (B) Coleta de dados dos indivíduos vivos em pé.

Para estimar a biomassa vegetal de cada uma das árvores vivas e mortas em pé, os valores das 
circunferências obtidas foram transformados em diâmetro, conforme a equação 1:

$$
D A P=C A P / \pi
$$

Em que: $\mathrm{DAP}=$ diâmetro do caule da árvore à altura do peito $\mathrm{CAP}=$ circunferência do caule da árvore à altura do peito $\pi=3,1416$

A biomassa vegetal aérea de cada indivíduo foi calculada pelo emprego da equação 2 , conforme Arevalo et al. (2002).

$$
B A=0,1184 \times D A P^{2,53}
$$

Em que: $B A=$ biomassa de árvores vivas e mortas em pé, 0,1184 = constante $\mathrm{DAP}=$ circunferência na altura do peito $\operatorname{DAP}(\mathrm{cm}), 2,53=$ constante.

Para calcular a quantidade de biomassa vegetal aérea por hectare, foi adaptada para essa pesquisa a equação 3, conforme Arevalo et al. (2002).

$$
B A V T(t / h a)=B T A V \times 0,04
$$

Em que: $B A V T=$ biomassa total de árvores vivos em $t / h a, B T A V=$ biomassa total na parcela de $20 \times 20 \mathrm{~m}, 0,04=$ fator de conversão para a parcela de $20 \times 20 \mathrm{~m}$.

\section{RESULTADOS E DISCUSSÃO}

A biomassa total em árvores vivas e mortas na Flona de Palmares foi estimada em $313.050 \mathrm{Mg} / \mathrm{ha}^{-1}$, no período de outubro e novembro de 2019. No total foram 218 indivíduos, entre eles estão algumas espécies e suas respectivas famílias: o caneleiro (Fabaceae), arruda (Rutaceae), cedro (Meliaceae), sapucarana (Lecythidaceae).

A tabela 1 mostra os resultados obtidos na quantificação da biomassa vegetal aérea, onde os maiores números de indivíduos estavam presentes nas parcelas 02 e 05 , por conseguinte obtiveram os maiores valores de biomassa, as duas parcelas estão localizadas na parte mais central e no fundo da Flona, com maiores altitudes e bem conservadas.

Tabela 1: Quantificação de biomassa arbórea da Flona de palmares Altos-PI, no período seco de 2019.

\begin{tabular}{lllll}
\hline \multirow{2}{*}{ Parcela } & \multicolumn{2}{l}{ Biomassa vegetal aérea } & & \\
\cline { 2 - 3 } & (Kg/árvore) & $\left(\mathrm{Mg} / \mathrm{ha}^{-1}\right)$ Total & 0,10 & Altura (média) \\
\hline 01 & 38,16 & 1.5264 & 0,19 & 8,44 \\
02 & 135,63 & 5.4252 & 0,13 & 11,73 \\
03 & 70,21 & 2.8084 & 0,19 & 11,30 \\
04 & 63,25 & 2.5300 & 0,11 & 10,52 \\
05 & 212,79 & 8.5116 & 0,13 & 8,55 \\
06 & 98,13 & 3.9252 & 0,10 & 9,01 \\
07 & 65,01 & 2.6004 & 0,12 & 11,82 \\
08 & 87,53 & 1.7506 & 0,24 & 10,26 \\
09 & 55,68 & 2.2272 & 1,13 & 13,22 \\
\hline Total & 826,39 & 313.050 & & 94,85 \\
\hline
\end{tabular}

Os valores das medias do DAP e da Altura das parcelas conforme a tabela 01, não influencia no resultado da biomassa dessa pesquisa, visto que a parcela 05 com maior valor de biomassa obteve uma média bem mais baixa do DAP considerando o valor das outras parcelas.

Vieira (2019) evidencia que a biomassa é diretamente proporcional ao DAP, quanto maior o DAP, maior o valor da biomassa. No entanto não quer dizer que a biomassa total será sempre maior, devido ao valor do DAP, considerando que a biomassa total é a soma de todas as biomassas arbóreas. Deste modo, leva-se em consideração a quantidade de árvores e consequentemente a biomassa arbórea das outras 
árvores.

A figura 4 mostra as porcentagens de biomassa das parcelas em que foi feito o estudo, a parcela 01 obteve a menor porcentagem isso pode ser justificado pelo fato dessa área ter obtido os menores números de indivíduos com o CAP maior que $15 \mathrm{~cm}$, o total de indivíduos dessa área foram apenas 18 , com altura entre $4 \mathrm{~m}$ e $11 \mathrm{~m}$, e CAP entre 15 e $71 \mathrm{~cm}$. Isso se reflete também para as outras áreas com baixa porcentagem de biomassa.

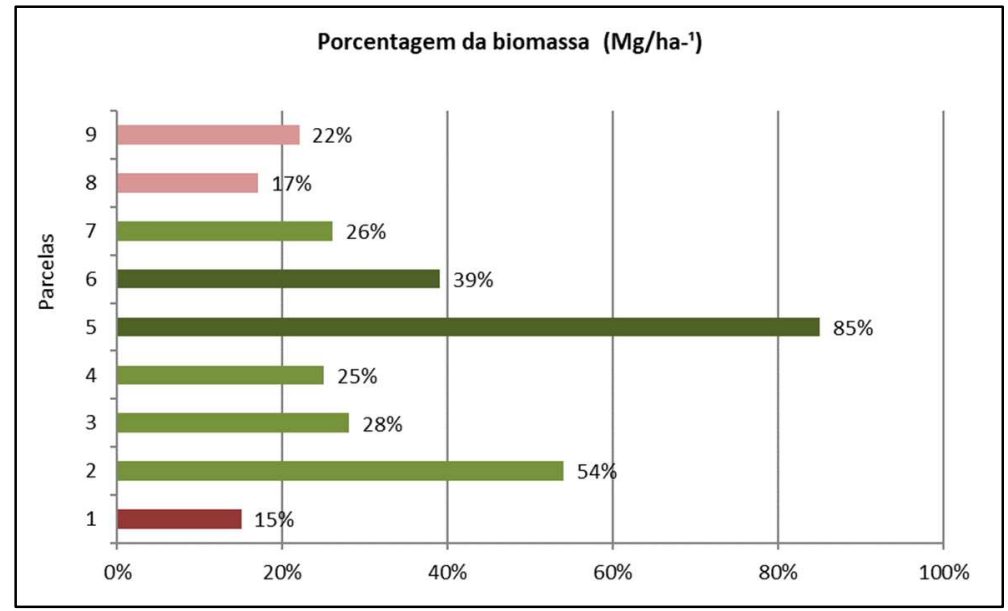

Figura 4: Porcentagem da biomassa das parcelas da Floresta Nacional de Palmares.

A parcela 05 que possui o maior valor de biomassa, isso se deve a quantidade de indivíduos em que foram coletados os dados, essa parcela obteve o maior número de indivíduos que no total foram 47 nessa área de diferentes espécies, tamanhos e tipologias. Pode ser observado no NDVI que é uma área com menos alterações na paisagem.

Viana et al. (2009) destaca que as imagens de satélite como o Landsat TM são bastante utilizadas no recolhimento de informações sobre vegetação, uma vez que a informação espectral apresenta uma forte correlação com a biomassa. Com isso é cada vez mais recente as estimativas de biomassa que se baseiam em métodos onde os índices de vegetação são utilizados.

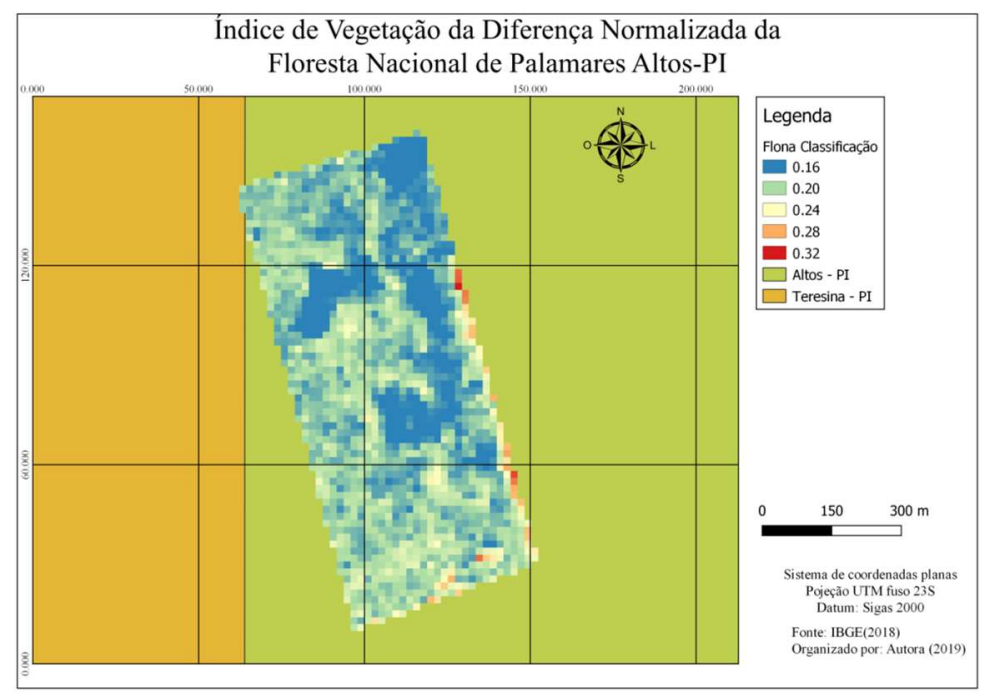

Figura 5: NDVI da Floresta Nacional de Palmares.

Com o NDVI é possível ver alterações da paisagem na parcela 01 e nas parcelas 08 e 09, o que explica 
os menores números de indivíduos arbóreos dessas áreas. Segundo Granado (2019), quanto maior a diferença na paisagem, maior será a variação na resposta espectral, captando maiores variações. A quantificação de biomassa em florestas é considerada uma importante ferramenta na avaliação da sua utilização e formação, pode ser empregada para diversos propósitos, como na avaliação da ciclagem de nutrientes, do sequestro de carbono, e, por conseguinte os impactos que possivelmente a retirada da biomassa possa vir a provocar no ambiente (VIRGENS et al., 2017).

Estudo sobre biomassa florestal na Caatinga tem oferecido informações relevantes (SILVA, 2019). Em um estudo feito por Virgens et al. (2017) em um trabalho realizado em uma Floresta Nacional Contendas do Sincorá, localizada no Município de Contendas do Sincorá, BA, onde avaliou os estoques e distribuição de biomassa da parte aérea de espécies da Caatinga arbórea, obteve total de biomassa seca foi estimado em $29,2 \mathrm{Mg}$ ha $(50,71 \mathrm{~kg} / \mathrm{arv})$.

Vieira (2019), ao estimar a Biomassa Vegetal Arbórea do Parque Municipal Américo Renné Giannetti em Belo Horizonte/MG, obteve um resultado de 909.618,41 kg para a biomassa aérea. Em um estudo feito por Nascimento (2019), estimativa da biomassa nas áreas de caatinga densa para a região semiárida de Pernambuco teve valor médio de $32,60 \mathrm{Mg} \mathrm{ha}^{-1}$.

As florestas naturais sofrem com processos de mudanças na estrutura e composição florística, devido a isso os padrões de variação espacial e temporal de biomassa estão em constante alteração. A biomassa é um indicador de produtividade e varia com a precipitação, temperatura e altitude (SANTOS et al., 2018). Diante disso é necessários estudos para entender a dinâmica e o estoque de biomassa florestal, e suas relações físico-químicas no ecossistema.

O resultado dessa pesquisa está um pouco semelhante à de outras pesquisas feitas em florestas, Lipinski et al. (2017) destaca que existem muitas diferenças nos estoques de biomassa em função de várias condições, como por exemplo, diferentes tipologias, ecossistemas, histórico de manejo florestal aplicado nessas áreas e também os diferentes métodos de quantificação e intensidade amostral.

A estimativa de biomassa de sistemas florestais permite amparar decisões vinculadas a atividades econômicas e ambientais, como políticas públicas, manejo florestal, estudos de ciclagem de nutrientes e ordenamento territorial (BOLFE et al., 2012). Miranda et al. (2019) afirma que há uma carência de estudos que indiquem, de fato, qual é a contribuição que as florestas têm em relação aos estoques de biomassa. No entanto segundo Hamilton et al. (2018) em termos de mudanças climáticas, são cada vez mais importantes conhecer os estoques de biomassa em distintos ambientes.

\section{CONCLUSÕES}

Os estudos referentes à biomassa florestal consideram em sua maioria o aspecto econômico deixando de lado os aspectos ecológicos, isso resulta em uma predominância de pesquisas com espécies e tipologias que possuam valor comercial, que não é o caso dessa pesquisa.

Com o NDVI foi possível ter uma visualização da vegetação da Flona, e assim fazer uma comparação com os resultados da biomassa, que foram coerentes com a imagem do NDVI. Essa pesquisa verificou que a 
biomassa vegetal aérea obteve uma média por hectare de $34.783 \mathrm{Mg} / \mathrm{ha}^{-1}$, e uma média de 91,82 Kg/árvore de biomassa. Vale ressaltar que existe uma crescente necessidade de estudos de biomassa vegetal aérea em florestas e áreas da caatinga.

\section{REFERÊNCIAS}

AREVALO, L. A.; ALEGRE, J. C.; VILCAHUAMAN, L. J. M.. Metodologia para estimar o estoque de carbono em diferentes sistemas de uso da terra. Colombo: Embrapa, 2002.

BOLFE, E. L.; BATISTELLA, M.; FERREIRA, M. C.. Correlação de variáveis espectrais e estoque de carbono da biomassa aérea de sistemas agroflorestais. Revista Pesquisa Agropecuária Tropical, Brasília, v.47, n.9, p.1261-1269, 2012.

FIORENTIN, L. D.; CORTE, A. P.; SANQUETTA, C. R.; BEHLING; A.. Quantificação e modelagem da biomassa e carbono da regeneração natural em área de floresta ombrófila mista. Revista Bras. Biom, São Paulo, v.33, n.2, p.251-267, 2015.

GRANADO, L. M. A.. Estimativa de biomassa e combustível em diferentes fitofisionomias do cerrado. Dissertação (Mestrado em Ciências Florestais) - Universidade de Brasília, Brasília, 2019.

HAMILTON, S. E.; FRIESS, D. A.. Global carbon stocks and potential emissions due to mangrove deforestation from 2000 to 2012. Revista Nature Climate Change, v.8, p.240244,2018

LIPINSKI, E. T.; CORTE, A. P.; SANQUETTA, C. R.; RODRIGUES, A. L.; MOGNON, F.; BEHLING, A.. Tree biomass and carbon dynamics between 1995-2012 in a Mountain Araucaria Forest. Revista Floresta, Curitiba, v.47, n.2, p.197-206, 2017 DOI: http://doi.org/10.5380/rf.v47i2.40024

MIRANDA, B. P.; RODERJAN, C. V.; BOTOSSO, P. C.; SANTOS, T. L.; ANDRADE, V. H. F.. Estimativa da biomassa aérea e carbono de ilex microdonta em floresta atlântica, Paraná, Brasil. Revista Biofix Scientific Journal, Paraná, v.4, n.1, p.58-63, 2019. DOI:

http://doi.org/dx.doi.org/10.5380/biofix.v4i1.63865

NASCIMENTO, D. M.. Desenvolvimento de um sistema de estimativa de biomassa vegetal através de sensores remotos para o semiárido pernambucano. Tese (Doutorado em Tecnologias Energéticas e Nucleares) - Universidade Federal de Pernambuco, Recife, 2019.

SALVADOR, S. M.; SCHUMACHER, M. V.; STAHL, J.; SANTOS, J. C.. Biomassa arbórea e de sub-bosque e da serapilheira acumulada em povoame ntos de Eucalyptus urophylla $x$ Eucalyptus grandi. Revista ENFLO Ecologia e Nutrição Florestal, Santa Maria, v.3, n.3, p.82-93, 2015.
SANQUETTA, C. R.; CORTE, A. P.; MOGNON, F.; MAAS, G. C. B.; RODRIGUES, A. L.. Estimativa de carbono individual para Araucaria angustifólia. Revista Pesquisa Agropecuária Tropical, Goiânia, v.44, n.1, p.1-8, 2014

SANTOS, F. G.; CAMARGO, P. B.; OLIVEIRA JUNIOR, R. C.. Estoque e dinâmica de biomassa arbórea em floresta ombrófila densa na flona tapajós: Amazônia oriental. Revista Ciência Florestal, Santa Maria, v.28, n.3, p.1049-1059, 2018. DOI: http://dx.doi.org/10.5902/1980509833388

SILVA, E. D. G.. Estimativa da biomassa e nutrientes em espécies arbóreas da caatinga, Rio Grande do Norte. Monografia (Bacharel em Engenharia Florestal) Universidade Federal Rural do Semiárido, Mossoró, 2019.

SNUC. Institui o Sistema Nacional de Unidades de Conservação da Natureza. Lei Federal n. 9.985 de 18 de julho de 2000. Brasília: DOU, 2000.

SOUSA, G. A.; SOARES, L. H. C.; NETO, C. F. C.. Plano de ação emergencial floresta nacional dos palmares. Governo do estado do Piauí, 2018.

VIANA, H.; LOPES, D.; ARANHA, J.. Predição de biomassa arbustiva lenhosa empregando dados de inventário e o índice de diferença normalizada extraído em imagens Landsat 5 TM. Revista Millenium, v.37, n.14, 2009

VIEIRA, J. A.. Estimativa do estoque de Carbono presente na biomassa vegetal arbórea do Parque Municipal Américo Renné Giannetti, Belo Horizonte/MG. Dissertação (Mestrado em Sustentabilidade e Tecnologia Ambiental) Instituto Federal de Educação, Ciência e Tecnologia de Minas Gerais, Bambuí, 2019.

VIRGENS, A. P.; BARRETO-GARCIA, P. A. B.; PAULA, A.; CARVALHO, F. F.; ARAGÃO, M. A.; MONROE, P. H. M. Biomassa de espécies florestais em área de caatinga arbórea. Revista Pesquisa Florestal Brasileira, Colombo, v.37, n.92, p.555-561, 2017. DOI: http://doi.org/10.4336/2017.pfb.37.92.1465

ZIEMMER, J. K.; BEHLING, A.; CORTE, A. P. D.. Quantificação da biomassa e dos teores de carbono de pteridófitas arborescentes em floresta ombrófila mista. Revista Biofix Scientific Journal, Paraná, v.1, n.1, p.60-73, 2016.

A CBPC - Companhia Brasileira de Produção Científica (CNPJ: 11.221.422/0001-03) detém os direitos materiais desta publicação. Os direitos referem-se à publicação do trabalho em qualquer parte do mundo, incluindo os direitos às renovaç̃oes, expansões e disseminações da contribuiç̃o, bem como outros direitos subsidiários. Todos os trabalhos publicados eletronicamente poderão posteriormente ser publicados em coletâneas impressas sob coordenação da Sustenere Publishing, da Companhia Brasileira de Produção Científica e seus parceiros autorizados. Os (as) autores (as) preservam os direitos autorais, mas não têm permissão para a publicação da contribuição em outro meio, impresso ou digital, em português ou em tradução. 